THE KURUME MEDICAL JOURNAL

Vol. 40, p. 59-63, 1993

\title{
Lymphocytic Alveolitis Associated with Asymptomatic Primary Biliary Cirrhosis
}

\author{
YOICHIRO ICHIKAWA, MASAZUMI SAISHO, HIDEYUKI KOGA, \\ SIRO TOKISAWA, NAOTO TOKUNAGA AND KOTARO OIZUMI \\ Department of Medicine, Kurume University School of Medicine, Kurume, 830 Japan
}

Received for publication April 13, 1993

\begin{abstract}
Summary: A case with biopsy-proven subclinical lymphocytic alveolitis in association with asymptomatic primary biliary cirrhosis is described.
\end{abstract}

Key words: primary biliary cirrhosis - lymphocytic alveolitis bronchoalveolar lavage (BAL) - lung biopsy - T lymphocyte

\section{Introduction}

Primary biliary cirrhosis $(\mathrm{PBC})$ is an auto-immune chronic active liver disease characterized by continuous and progressive inflammation of the liver and liver cell destruction (Eggink et al. 1982). The association of $\mathrm{PBC}$ with auto-immune diseases such as Sjögren's syndrome, Hashimoto's disease and renal tubular acidosis has been reported (Yap et al. 1980). However, there have been few descriptions of the association of PBC and pulmonary involvement.

\section{Case Report}

On 20 July 1987, a 53 year-old woman was admitted to Kurume University Hospital with common cold-like symptoms such as cough, sputum production and low grade fever of several days' duration. She was not a smoker and her past medical history revealed no significant findings. A physical examination revealed a diffuse goiter in the neck. However, there were no rales in the chest, clubbing of the fingers, cyanosis, hepatomegaly, jaundice or lymphadenopathy.

The following laboratory data were obtained. Liver function tests: alkaline phosphatase, 48.0 King-Armstrong U/1 (normal $<10.0$ ), SGOT, 43.1 U/1 (normal $\langle 40.0$ ); $\gamma$-GTP, $249 \mathrm{mIU} / 1$ (normal $\langle 40$ ); LAP, 521 Goldbarg-Rutenberg U/1 (normal 100-200). The total bilirubin, SGPT, LDH and total cholesterol were normal. Thyroid function tests were normal. Serological tests: anti-mitochondrial antibody was positive at a titer of $1: 160$; anti-microsome antibody was positive at a titer of 1:1600; anti-nuclear antibody, anti-DNA antibody and rheumatoid latex fixation tests were all negative. The immunoglobulin $\mathrm{M}$ was $435 \mathrm{mg} / \mathrm{dl}$ (normal 80-200). The immunoglobulin $\mathrm{G}$ and immunoglobulin A were normal. Bacterial and fungal cultures of the sputum were negative. The chest roentgenogram was nearly normal. Pulmonary function tests were also nearly normal with a VC of $2.5 \mathrm{~L}$, a $\mathrm{VC} / \mathrm{VC}$ predicted of $96.5 \%$, an $\mathrm{FEV}_{1}$ of $1.9 \mathrm{~L}$, and an $\mathrm{FEV}_{1} / \mathrm{FVC}$ of

Reprint requests should be addressed to: Yoichiro Ichikawa, MD, The First Department of Medicine, Kurume University School of Medicine, 67 Asahi-machi, Kurume, 830 Japan. 


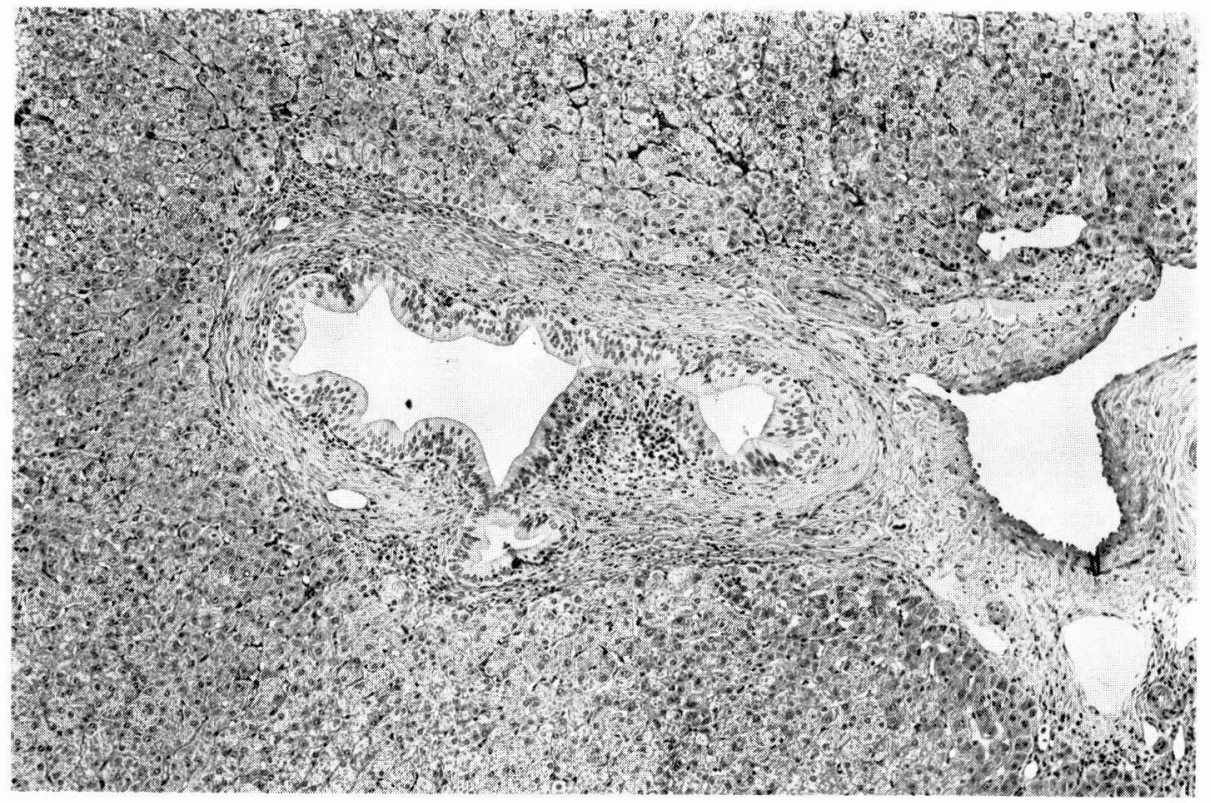

Fig. 1A. The septal and larger interlobular bile ducts were damaged and surrounded by an infiltrate of lymphocytes, epithelioid cells, and plasma cells. (Hematoxylin and eosin, original magnification, $\times 50$ ).

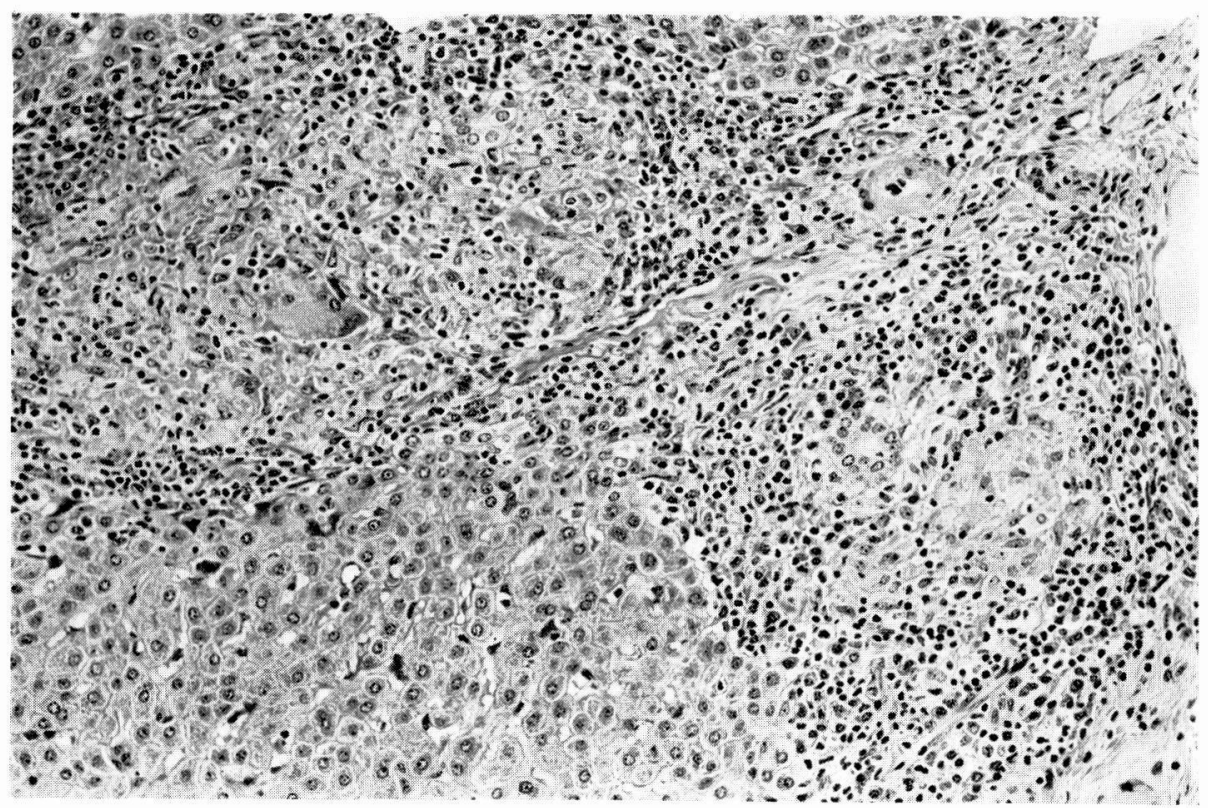

Fig. 1B. A liver biopsy specimen showing giant cell granulomas with mononuclear cells infiltrating into the portal tracts (Hematoxylin and eosin, original magnification, $\times 100)$. 




Fig. 2. A lung biopsy specimen from a trans-bronchial biopsy showing slight infiltration with mononuclear cells into the bronchiolovascular sheath and lung interstitium (Hematoxylin and eosin, original magnification,

$\times 100)$.

79. $1 \%$. The Dco was $92.4 \%$ of the predicted value. However, atrial blood gases on room air showed: $\mathrm{pH} 7.430, \mathrm{PCO}_{2} 42.8$ $\mathrm{mmHg}, \mathrm{PO}_{2} 76.8 \mathrm{mmHg}$. She spontaneously made favorable progress after admission, and one week later she was free of pulmonary symptoms.

On 20 August 1987, an open liver biopsy was performed to diagnose the liver function abnormalities. The surface of the liver was nodular in appearance. The liver biopsy specimens had mononuclear cell infiltration, periportal fibrosis and granulomatous changes within the portal tracts. The septal and larger interlobular bile ducts were damaged and surrounded by an infiltrate of lymphocytes, epithelioid cells, and plasma cells (Fig. 1A). Granulomas including Langhans' giant cells were present in the portal tracts and central zones (Fig. 1B). These pathological features were compatible with PBC stage I. At that time, the diagnosis was asymptomatic $\mathrm{PBC}$ associated with probable Hashimoto's disease.

On 11 September 1987, bronchoalveolar lavage (BAL) was performed. Cellular analysis of the BAL fluid revealed $52 \%$ alveolar macrophages, $40 \%$ lymphocytes, $7 \%$ neutrophils and $1 \%$ eosinophils. The lymphocytes recovered by BAL consisted of $24.7 \%$ helper $\mathrm{T}$ cells $\left(\mathrm{CD}^{+}\right)$and $19.1 \%$ supressor $\mathrm{T}$ cells $\left(\mathrm{CD}^{+}\right)$, and the $\mathrm{CD} 4^{+} /$ $\mathrm{CD}^{+}$ratio was 1.3 . One week later, a transbronchial lung biopsy was performed to further investigate the pulmonary involvement. The lung biopsy specimens revealed a slight infiltration of mononuclear cells in the bronchiolovascular sheath and lung interstitium (Fig. 2). The patient made favorable progress without clinical symptoms and had neither a worsening of liver function tests nor chest X-ray abnormalities during an approximately three year period after the discharge. 


\section{Discussion}

The development of bronchoalveolar lavage studies has increased our knowledge of alveolitis in diffuse pulmonary diseases. Alveolitis is considered to be a non-specific inflammation consisting of an accumulation of immune and inflammatory cells within alveolar structures with or without morphological lesions. Inflammatory alveolitis does not necessarily lead to a clinically significant interstitial pulmonary process.

In this case, lymphocytosis in the lower respiratory tract and infiltration of mononuclear cells in the lung interstitium were revealed by BAL and transbronchial lung biopsy. However, pulmonary function tests and a chest roentgenogram were nearly normal, except for the presence of a low grade hypoxemia. Furthermore, diseases that induce pulmonary lymphocytosis or lymphocytic alveolitis, such as sarcoidosis, hypersensitivity pneumonitis or other granulomatous lung diseases, were excluded clinically. These findings indicate that a subclinical lymphocytic alveolitis may occur in a patient with asymptomatic PBC.

Pulmonary involvement in $\mathrm{PBC}$ is uncommon and has rarely been reported (Turner-Warwick, 1968; Golding et al. 1973). In the previous reports, pulmonary involvement in $\mathrm{PBC}$ included pathological findings of two types. One was lung parenchymal granulomas similar to those in sarcoidosis and the other was fibrosing alveolitis or interstitial pneumonia (Fox et al. 1969; Stanley et al. 1972; Weissman and Becker, 1983; Maddrey et al. 1985). PBC and sarcoidosis are characterized by the development of granulomatous inflammation in multisystem organs, and similarities in the two diseases have been pointed out previously (Fox et al. 1969; Stanley et al. 1972). In view of the presence of granulomas in the liver biopsy specimens and the con- comitant lymphocytic alveolitis, sarcoidosis was considered as a differential diagnosis. However, infiltration of the liver by a sarcoid granuloma is usually manifested by minor abnormalities of the liver function tests; and the granulomas can be found not only in or near the portal tracts, but also in the lobules histologically. Furthermore, the increased serum IgM level, the absence of bilateral hilar lymphadenopathy and normal levels of serum angiotensin converting enzyme were not readily compatible with sarcoidosis. These results indicated that sarcoidosis was distinguishable from PBC. Fibrosing alveolitis was described in patients with chronic liver diseases by Turner-Warwick (1968). Later, Golding et al. (1873). reported that six of eight PBC patients with impairment of gas transfer had abnormal chest roentgenograms and two had a mixed pattern of the usual interstitial and desquamative types of pneumonia. Lymphoid interstitial pneumonia or acute interstitial pneumonia has also been found in patients with PBC (Yap et al. 1980; Weissman and Becker, 1983).

In 1981, Rodriguez-Roisin et al. (1981) reported that gas transfer was reduced in patients with $\mathrm{PBC}$ associated with Sjögren's syndrome and in patients with sicca complex, and that the respiratory, clinical, and functional abnormalities found in $\mathrm{PBC}$ are related to the presence of an associated Sjögren's syndrome. However, our patient had PBC that was associated with probable Hashimoto's disease, but not associated with Sjögren's syndrome.

The pathogenesis of $\mathrm{PBC}$ and fibrosing alveolitis remains unknown; however, both diseases are characterized by overlapping multi-organ system involvement, immunological abnormalities such as the presence of circulating immune complexes and histological findings (Yap et al. 1980). Accordingly, the association of $\mathrm{PBC}$ and 
fibrosing alveolitis may not be accidental and may result from a common pathogenetic mechanism. Wallert et al. (1986) reported that subclinical alveolar inflammation consisting of $\mathrm{T}$-lymphocytes and activated macrophages was present in a high proportion of patients with PBC who had normal chest roentgenograms. They also stated that the subclinical alveolitis was not simply secondary to the extent of the disease, because there was no correlation between the $\mathrm{BAL}$ abnormalities and the histologic stage of $\mathrm{PBC}$ or bilirubin concentration.

Although our patient had asymptomatic PBC, lymphocytic alveolitis was demonstrated. This indicates that a subclinical alveolitis may occur at an early stage of $\mathrm{PBC}$ and that lymphocytic alveolitis with multi-organ system involvement in PBC and fibrosing alveolitis may have a common pathogenetic mechanism.

At present, the role of pulmonary lymphocytosis in $\mathrm{PBC}$ remains unknown and the prognosis of subclinical lymphocytic alveolitis in PBC is unclear. However, many cases of subclinical lymphocytic alveolitis in PBC may not become aggravated because overt pulmonary involvement rarely has been reported in PBC. Further studies are required to determine the functional status of lymphocytes in the lungs of patients with PBC.

Acknowledgments: The authors would like to thank Satoko Nakano for her secretarial as sistance in the preparation of this manuscript.

\section{References}

Eggink, H.F., Houthoff, H. J., Huitema, S., Gips, C.H. and Poppema, S. (1982). Cellular and humoral immune reactions in chronic active liver disease. I. Lymphocyte subsets in liver biopsies of patients with untreated idiopathic autoimmune hepatitis, chronic active hepatitis B and primary biliary cirrhosis. Clin. Exp. Immunol. 50, 17-24.

Fox, R.A., James, D.G., Scheuer, P.J. and Sharma, O. (1969). Impaired delayed hypersensitivity in primary biliary cirrhosis. Lancet 1, 959-962.

Golding, P.L., Smith, M. and Williams, R. (1973). Multisystem involvement in chronic liver disease. Am. J. Med. 55, 772-782.

Maddrey, W.C., Sha, E. and Keeffe, E. (1985). When sarcoidosis overlaps primary biliary cirrhosis. J. Respir. Dis. 6, 41-48.

Rodrignez-Roisin, R., Pares, A., Bruguera, M., Coll, J., Picado, C. et al. (1981). Pulmonary involvement in primary biliary cirrhosis. Thorax 36, 208-212.

Stanley, N.N., Fox, R.A., Whimster, W.F., Sherlock, S. and James, D.G. (1972). Primary biliary cirrhosis or sarcoidosis-or both. N. Engl. J. Med. 287, 1282-1284.

TURner-WARWICK, M. (1968). Fibrosing alveolitis and chronic liver disease. Q. J. Med. (New Series) 37, 133-149.

W allaert, B., Bonniere, P., Rrin, L., Cortot, A., Tonnel, A.B. et al. (1986). Primary biliary cirrhosis. Subclinical inflammatory alveolitis in patients with normal chest roentgenograms. Chest 90, 842-848.

Weissman, E. and Becker, N.H. (1983). Interstitial lung disease in primary biliary cirrhosis. Am. J. Med. Sci. 285, 21-27.

YAP, S.H., Schillings, P.H.M. and VAN Tongeren, J.H.M. (1980). Acute interstitial pneumonia (fibrosing alveolitis) in a patient with primary biliary cirrhosis. Neth. J. Med. 23, 111-116. 